\title{
Comparative analysis of codon usage patterns in chloroplast genomes of five Miscanthus species and related species
}

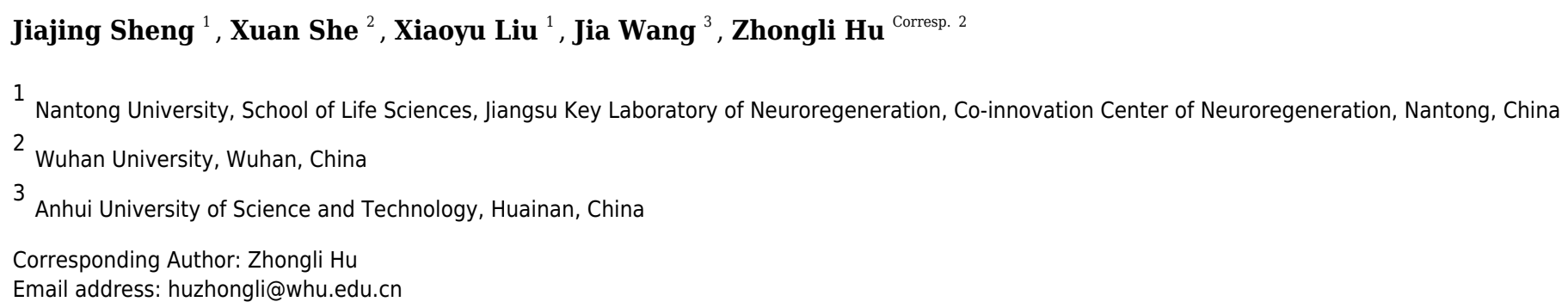

Miscanthus is not only a perennial fiber biomass crop, but also valuable breeding resource for its low-nutrient requirements, photosynthetic efficiency and strong adaptability to environment. In the present study, the codon usage patterns of five different Miscanthus plants and other two related species were systematically analyzed. The results indicated that the cp genomes of the seven representative species were preference to $A / T$ bases and A/T-ending codons. In addition, 21 common high-frequency codons and 4-11 optimal codons were detected in the seven chloroplast genomes. The results of ENc-plot, PR2-plot and neutrality analysis revealed the codon usage patterns of the seven chloroplast genomes are influenced by multiple factors, in which nature selection is the main influencing factor. Comparative analysis of the codon usage frequencies between the seven representative species and four model organisms suggested that Arabidopsis thaliana, Populus trichocarpa and Saccharomyces cerevisiae could be considered as preferential appropriate exogenous expression receptors. These results might not only provide important reference information for evolutionary analysis, but also shed light on the way to improve the expression efficiency of exogenous gene in transgenic research based on codon optimization. 


\title{
1 Comparative analysis of codon usage patterns in
}

2 chloroplast genomes of five Miscanthus species and

3 related species

4

5

6

7

8

9

10

11

12

13

14

15

16

17

18

19

20

21

22

23

24

25

26

27

28

29

30

31

32

33

34

35

36

37

38

\author{
Jiajing Sheng1, Xuan She2, Xiaoyu Liu1, Jia Wang3, Zhongli Hu2* \\ 1 School of Life Sciences, Jiangsu Key Laboratory of Neuroregeneration, Co-innovation Center of Neuroregeneration, Nantong University, \\ Nantong, 226019, PR China \\ 2 State Key Laboratory of Hybrid Rice, Lotus Engineering Research Center of Hubei Province, College of Life Sciences, Wuhan University, \\ Wuhan, 430072, PR China \\ 3 Key Laboratory of Industrial Dust Prevention and Control \& Occupational Safety and Health of the Ministry of Education, Anhui University of \\ Science and Technology, Huainan, 232001 China \\ Corresponding Author: \\ Zhongli $\mathrm{Hu}$ \\ State Key Laboratory of Hybrid Rice, Lotus Engineering Research Center of Hubei Province, \\ College of Life Sciences, Wuhan University, Wuhan, 430072, PR China \\ Email address: huzhongli@whu.edu.cn
}

\section{Abstract}

Miscanthus is not only a perennial fiber biomass crop, but also valuable breeding resource for its low-nutrient requirements, photosynthetic efficiency and strong adaptability to a changing environment. In the present study, the codon usage patterns of five different Miscanthus species and that of two other related species were systematically analyzed. The results indicated that the chloroplast genomes of the seven representative species showed preference for A/T bases and A/T-ending codons. In addition, 21 common high-frequency codons and 4 to 11 optimal codons were detected in the seven chloroplast genomes. The results of ENc-plot, PR2-plot and neutrality analysis revealed the codon usage patterns of the seven chloroplast genomes are influenced by multiple factors, in which natural selection is the main influencing factor. Comparative analysis of the codon usage frequencies between the seven representative species and four model organisms suggested that Arabidopsis thaliana, Populus trichocarpa and Saccharomyces cerevisiae could be considered as preferential heterologous expression hosts. These results might not only provide important reference information for evolutionary analysis, but also shed light on the way to improve the expression efficiency of exogenous gene in transgenic research based on codon optimization.

\section{Introduction}

Miscanthus Andersson (Poaceae) is a C4 photosynthetic plant, which have been widely investigated as a potential second-generation bio-energy crop (Barling et al. 2013). The genus Miscanthus includes approximately 20 species, which could be classified into Miscanthus clades 
40

41

42

43

44

45

46

47

48

49

50

51

52

53

54

55

56

57

58

59

60

61

62

63

64

65

66

67

68

69

70

71

72

73

74

75

76

77

78

79

and Triarrhena clades (Ge et al. 2017). China is the biological diversity center of Miscanthus species, of which Miscanthus lutarioriparius L.Liou (M. lutarioriparius), Miscanthus sinensis Andersson (M. sinensis), Miscanthus sacchariflorus (Maxim.) Nakai (M. sacchariflorus) and Miscanthus floridulus (Lab.) Warb. ex Schum. et Laut (M. floridulus) are the four most widely distributed species. In addition to being bioenergy plants, Miscanthus species possess extensive breeding values due to their extremely advantageous agricultural characteristics, such as high photosynthetic efficiency, cold tolerance and an extensive ability to adapt to environmental change (Vermerris 2008). Currently, the research focus of Miscanthus species is to utilize it as a promising genetic resource (Clark et al. 2015; Zhang et al. 2013). The more diverse genetic resources available, the more likely it is that scientific research will be able to comprehend the adaptation, evolution and utilization of these significant economic crops.

Chloroplasts (cp) are key plastids involved in multifunctional processes of the plant cell, including photosynthesis, carbon fixation, starch storage, nitrogen metabolism, fatty acid and nucleic acid synthesis (Jarvis \& López-Juez 2013; Nielsen et al. 2016). Typically, cp genomes (cpDNAs) possess a small size, conserved gene content and large copy numbers, which have been extensively used as valuable source for evolution analysis and plastid engineering (Amiryousefi et al. 2018; Ravi et al. 2008; Yan et al. 2019). The lack of genomic resources of the Miscanthus species has hindered the adequate understanding of their diversity traits (Chae et al. 2014; Sheng et al. 2016) The low expression efficiency of an exogenous gene may limit the research progress on the functional studies of Miscanthus and their related species (Wu et al. 2021). Stemming from the rapid development of cp engineering, plasmid DNA have been transferred into the cpDNA of a variety of plants, such as Nicotiana tabacum, Manihot esculenta Crantz and Eruca sativa Mill (Havaux et al. 2003; Khodakovskaya et al. 2006; Kwak et al. 2019). Recently, the cpDNA of some Miscanthus species have been made available in the National Center for Biotechnology Information (NCBI) database (Sheng et al. 2021). These complete cpDNA sequences of Miscanthus species can be used for studying population genetics, evolution analysis and plastid engineering (Amiryousefi et al. 2018; Yan et al. 2019).

Codon usage bias refers to the variations on the usage frequencies of synonymous codons (Plotkin \& Kudla 2011). The pattern of codon usage could be caused by multi-factors during the process of genome and gene evolution, including natural selection, compositional mutation mode, translational selection, gene length, tRNA abundance and mRNA secondary structure (Liu et al. 2004; Pop et al. 2014; Quax et al. 2015; Tuller et al. 2010). The studies of codon preference can not only reveal the evolutionary rules between genes in a species or related species, but also improve the expression efficiency of exogenous sequences in transgenic research by codon optimization. Recently, the applicability of codon optimization in the cpDNA have been determined for many vascular plants, including Poaceae (Gramineae) (Zhang et al. 2012), Cinnamomum camphorn (L.) presl (Camphor tree ) (Chen et al. 2017), Fragaria $\times$ ananassa Duch (strawberry) (Cheng et al. 2017) and Solanum tuberosum L. (potato) (Zhang et al. 2018). However, the codon usage pattern of cpDNA in Miscanthus and related species has not been fully elucidated.

Peer) reviewing PDF | (2021:03:58914:3:1:NEW 11 Aug 2021) 
80

81

82

83

84

85

86

87

88

89

90

91

92

93

94

95

96

97

98

99

100

101

102

103

104

105

106

107

108

109

110

111

112

113

114

115

116

117

118

In this study, the codon usage patterns of the cpDNA of seven Miscanthus and related species, including M. sinensis, Miscanthus transmorrisonensis Hayata (M. transmorrisonensis), M. floridulus, M. sacchariflorus, Miscanthus x giganteus (M. x giganteus), Sorghum bicolor and Saccharum spontaneum were systematically analyzed based on the previously published genome-wide data. Among them, M. sinensis, M. transmorrisonensis and M. floridulus belong to the Miscanthus clades under the Miscanthus species, M. sacchariflorus belongs to the Triarrhena clades under the Miscanthus species, and M. x giganteus is a natural triploid hybrid. According to our previous studies, Miscanthus are most closely related to Sorghum bicolor (L.) Moench and Saccharum L (Sheng et al. 2021; Sheng et al. 2017). In addition, the codon usage bias of these seven species was compared with the other four model species including Populus trichocarpa Torr \& Gray, Escherichia coli, Arabidopsis thaliana (L.) Heynh and Saccharomyces cerevisiae. Miscanthus are not only potential bio-energy crops, but also forms an excellent breeding resources. So, it is of interest to understand the codon usage of Miscanthus for better utilization of Miscanthus and related resources as germplasm resources. Here, we revealed the codon usage patterns of the Miscanthus and related species and determined optimal codons for cpDNA genetic engineering. The results in the current study will not only provide insight into genetic evolution studies, but also provide a reference for selecting appropriate heterologous expression hosts to improve the gene expression of Miscanthus plants by optimizing codon.

\section{Materials \& Methods}

\section{Genomes and sequences selection}

The complete cpDNAs of $M$. floridulus (NC_035750.1), M. sacchariflorus (NC_028720.1), $M$. sinensis (NC_028721.1), $M . \quad x$ giganteus $\quad\left(\mathrm{NC}_{0}\right.$ 035753.1), $M . \quad$ transmorrisonensis (NC_035752.1), Sorghum bicolor (NC_008602), Saccharum spontaneum (NC_034802.1) with gene annotation were downloaded from the NCBI GeneBank database. The number of raw sequence coding for amino acids in protein (CDS) of above seven species was 106, 122, 122, 106, 106, 84 and 76 respectively (Table 1). To avoid sampling bias, the CDS sequences were screened from genome-wide data by python scripts (https://github.com/shexuan/codon analysis) according to the following principles: (1) CDS contains initiation codon (ATG), termination codons (TAA, TAG or TGA) and without intermediate stop codons in the sequences; (2) the number of bases in each CDS must be the fold of three (3) the length of the sequence of CDS should be $\geqslant 300$ bp (Wright 1990; Zhang 2007). After filtration, the CDS number, the base composition at the first/second/third site of codons (GC1/GC2/GC3) and average GC, as well as the total amino acids encoded by each CDS were calculated.

\section{Analysis of relative synonymous codon usage (RSCU) and relative synonymous codon usage frequency (RFSC)}

Relative synonymous codon usage value of a codon (the number of codon occurrences in a gene divided by the number of codon appearances expected under the same codon usage) is the ratio 
119 of its actual frequency of utilization to the expected usage frequency without bias. The RSCU 120 was calculated as Eq. (1):

121

$122 \mathrm{RSCU}=\frac{x_{i j}}{\sum_{j}^{N_{i}} x_{i j}} n_{i}$

123 Where $x_{i j}$ represents the frequency of codon $j$ encoding the $i$ th amino acid, and $n_{i}$ represents the

124

125

126

127

128

129

130

131

132

133

134

135

136

137

138

139

140

141

142

143

144

145

146

147

148

149

150

151

152

153

154

155

156 number of synonymous codon encoding the $i$ th amino acid (Sharp, 1986). If the RSCU value of a codon is equal to 1 , the codon is used without bias, whereas a RSCU value greater than 1 reflects a significant codon usage bias (Sharp, 1987).

The RFSC value refers to the proportion of the actually observed number of a codon in the number of all synonymous codons. The RFSC were calculated using Eq. (2):

$\mathrm{RFSC}=\frac{x_{i j}}{\sum_{j}^{N} x_{i j}}$

Where $\mathrm{x}_{\mathrm{ij}}$ represents the frequency of codon $\mathrm{j}$ encoding for the $\mathrm{I}$ th amino acid. The highfrequency codon was screened based on the results of RFSC in all codon. The screening principles were as follows: the RFSC $>60 \%$ of one codon; or the RFSC of a codon exceeds the average frequency of synonymous codon by 0.5 times (Zhou et al. 2007).

\section{Determination of optimal codons}

The effective number of codons (ENC) can be applied to describe the extent of deviation of codon usage from the random selection, which reflects the degree of unbalanced use of synonymous codon in genes. The ENC value range from 20 (each amino acid uses only one synonymous codon) to 61 (Each synonymous codon is equally used), which is inversely proportional to the codon bias (Wright 1990). The ENC value in each species was calculated by CodonW software and then $10 \%$ of the CDS with remarkably high and low expression levels were filtered out according to the ENC value. The RSCU of each codon was obtained from the sequence files of the high and low groups according to the cusp function of emboss (https://www.bioinformatics.nl/emboss-explorer/). Optimal codons were determined by $\Delta$ RSCU method. Specifically, the average RSCU values of the two amino acid groups were computed and subtract subsequently ( $\triangle \mathrm{RSCU})$. The codon will be identified as the optimal codon through comparing the high and low group of the same codon $\triangle \mathrm{RSCU}(>0.08)$ and RSCU value (high group $>1$, low group $<1)$ (Romero et al. 2000).

\section{Comparative analysis of codon usage frequency}

The ratio of codon usage frequency is one indicator of codon usage bias among species. To further explore the codon usage patterns in the seven species of Miscanthus and their relatives, codon usage bias data of four model species including Escherichia coli (http://ww w.kazusa.or.jp/codon/cgi-bin/showcodon.cgi?species=199310); Saccharomyces cerevisiae (http://www.kazusa.or.jp/codon/cgi-bin/showcodon.cgi?species=4932); Populus trichocarpa (http://www.kazusa.or.jp/codon/cgi-bin/showcodon.cgi?species=3694) and Arabidopsis thaliana 
157 (http://www.kazusa.or.jp/codon/cgi-bin/showcodon.cgi?species=3702), which have been used as

158 the most common heterologous expression hosts were downloaded from the Codon Usage 159 Database. Subsequently, the codon usage frequencies of the seven species in this study were 160 compared with the above four model organisms. When the ratio is $\geqslant 2$ or $\leqslant 0.5$, it suggests that 161 the codon bias difference between the two organisms is significant, whereas other values outside 162 163 of this range represent a lack of significance (Pan et al. 2013).

168

169

170

171

172

173

174

175

176

177

178

179

180

181

182

183

184

185

186

187

188

189

190

191

192

193

194

195

196

\section{Analysis of ENC-plot}

The proportion of $\mathrm{G}$ and $\mathrm{C}$ content at the third position of a codon to the total number of gene bases are defined as GC3s. ENC-plot is plotted with ENC values as ordinate and GC3 value as abscissa, which can be used to analyze the codon usage characteristics of each gene and to explore the relevance between gene base component and codon preference (Wright 1990). ENC values are located on or near the expected curve, when mutation pressure plays a key role in the formation of codon usage patterns. Conversely, when the use of a codon is constrained by natural selection, the ENC value will be well below the prospective curve (Wright 1990).

\section{PR2-plot analysis}

In $\mathrm{G} 3 /(\mathrm{G} 3+\mathrm{C} 3)$ as the abscisic and $\mathrm{A} 3 /(\mathrm{A} 3+\mathrm{T} 3)$ as the ordinate graphic mapping (PR2-plot), which is performed to explore the composition of the four bases at the third nucleotide position of each codon (Sueoka 1999; Sueoka 1995). The pattern of splashes around the central spot $(A=T, C=G)$ indicate the extent and orientation of the base offset.

\section{Analysis of Neutrality plot}

Neutrality analysis is used to exploring the degree of impact between natural selection and mutation pressure on the mode of codon usage (Sueoka 1988). GC12 indicates the mean GC content at the first and second nucleotide positions of the codon, while GC3 represents the GC content of the third site. GC content at the third nucleotide position of a codon was counted post eliminating the Codon Met (ATG) and Trp (TGG). GC3 was counted post the eliminating of the three stop codons (TAA, TAG and TGA) and three codons (ATT, ATC and ATA) of Ile (Sueoka 1988). Both the GC12 and GC3 values of the seven cpDNAs were counted by Python scripts (https://github.com/shexuan/codon_analysis). If the gradient of the curve regression is 0 , indicating that there is no impact of mutation pressure. Gradient 1 represents complete neutrality, which describes that codon usage preference is completely influenced by mutation pressure (Sueoka 1988).

\section{Correspondence analysis of codon usage}

The variations of codon usage in the seven analyzed cpDNAs were investigated based on the correspondence analyses (COA) using CodonW (Anue et al. 2019). The usage patterns of 59 codons (exincluding Met, Trp and three termination codons) were compared and all genes can be embeded into a 59-dimension hyperspace, in which each dimension corresponds to the 
197

198

199

200

201

202

203

204

205

206

207

208

209

210

211

212

213

214

215

216

217

218

219

220

221

222

223

224

225

226

227

228

229

230

231

232

233

234

235

236

synonymous codon usage of the gene (Xiang et al. 2015). Therefore, the major trends (Axis 1) of these axes in the 59-dimensional hyperspace can be used to determine the maximum fraction of genetic variation, indicating the major sources of codon usage variation. In addition, according to the results of COA, the correlation index between Axis1 and codon usage exponent, including the GC content of codons, GC3s, codon adaptation index (CAI) and the total numbers of amino acids in the encoded polypeptide (L_aa) were computed by python scripts package (https:/github.com/shexuan/ codon_analysis). CAI value is widely applied to assess gene expression levels, ranging from 0 to 1 . Specifically, the larger the CAI value is, the stronger the codon usage preference is, and vice versa (Sharp \& Li 1986).

\section{Results}

\section{Characteristics of codon usage bias}

\section{Analysis of codon base composition}

The screened CDSs numbers processed by Python scripts are 65, 64, 64, 64, 64, 48 and 52 for $M$. floridulus, M. giganteus, M. sacchariflorus, M. sinensis, M. transmorrisonensis, Saccharum spontaneum and Sorghum bicolor respectively. In addition, the GC contents of three positions of codons (GC1, GC2, GC3) were calculated respectively (Table 1). It was found that the contents of $\mathrm{GC}$ at all three sites and the average GC content (GC123) were all less than 0.5, which indicated the seven analyzed cpDNAs were prone to use $\mathrm{A} / \mathrm{T}$ bases and $\mathrm{A} / \mathrm{T}$-ending codons (Table 1). Specifically, the mean GC content of three sites in M. floridulus, M. giganteus and $M$. transmorrisonensis was determined to equal the same value $(0.375)$, as well as a matching value determined for M. sacchariflorus and M. sinensis (0.393), but slightly different in Saccharum spontaneum (0.391) and Sorghum bicolor (0.39) (Table 1). Furthermore, the distribution trend of GC content was $\mathrm{GC} 1>\mathrm{GC} 2>\mathrm{GC} 3$, indicating that $\mathrm{GC}$ was not evenly distributed in the three positions of a codon of the seven assessed species. In summary, the codon usages of GC content in these seven cpDNAs were similar and were biased towards $\mathrm{A} / \mathrm{T}$ bases.

Table 1 Genomic features of $\mathrm{cp}$ genomes of the seven Miscanthus and related species (the total number of amino acids: $\mathrm{L}_{-}$aa ; the GC content at the first, second and third codon positions: GC1, GC2and GC3; average GC at three locations: GC123).

\section{RSCU and RFSC}

The cp genomes of the seven Miscanthus and related species have 30 common codons (RSCU > 1) with 28 codons ending with the nucleotides A/T (93.3\%) (Table S1). Therefore, almost the majority of codons of the seven plants species analyzed (RSCU $>1$ ) are likely to end with A/T. The variation ranges in the RSCU values were close in the seven cpDNAs, i.e., $0.31-1.93$ in $M$. floridulus, M. giganteus and M. transmorrisonensis, 0.32-1.94 in M. sacchariflorus and $M$. sinensis, 0.32- 2.01 in Saccharum spontaneum and 0.33-2.04 in Sorghum bicolor, respectively 
237 (Table S1). In addition, the maximum and the minimum RSCU values belonged to TTA and 238 CTG which encode Leu, indicating the vitally positive bias. Furthermore, the pattern of codon

239

240

241

242

243

244

245

246

247

248

249

250

251

252

253

254

255

256

257

258

259

260

261

262

263

264

265

266

267

268

269

270

271

272

273

274

275

276

usage were summarized in the seven Miscanthus and related species (Figure 1). Specifically, the high-frequency codons of seven Miscanthus and related species possess strong common base and share a total of 21 high-frequency codons (Table S1). Furthemore, Saccharum spontaneum and Sorghum bicolor were determined to possess two more high-frequency codons, specifically CGT and TAA, than the other five Miscanthus species.

Figure 1. Codon content in all protein-coding genes of the seven Miscanthus and related chloroplast genomes. The histogram of each amino acid indicated codon usage within the seven species.

(From left to right: M. floridulus, M. giganteus, M. sacchariflorus, M. sinensis, M. transmorrisonensis, Saccharum spontaneum and Sorghum bicolor).

\section{Determination of optimal codons}

The ENC values of each CDS were ranked and $10 \%$ of genes from both ends were selected to establish high and low expression gene banks respectively. The RSCU values and $\triangle \mathrm{RSCU}$ values in the two expression libraries were calculated and are listed in Supplementary Table S2. According to the values of $\triangle \mathrm{RSCU}$, the optimal codons in the seven assessed species were determined as follows (Table 2).

Table 2 Optimal codons in chloroplast genomes of the seven Miscanthus and related species.

\section{Codon usage frequency}

The codon usage frequencies of the seven cpDNAs were compared with four model species including Escherichia coli, Saccharomyces cerevisiae, Arabidopsis thaliana and Populus trichocarpa (Table S3). The Results of our analyses indicated that there is little divergence in the codon usage frequencies among the seven assessed plant species with Saccharomyces cerevisiae, Arabidopsis thaliana and Populus trichocarpa, have 9 to 11 (accounting for $14.1 \%-17.2 \%$ of total codons), 13 to $14(20.3 \%-21.9 \%), 12$ to $13(18.8 \%-20.3 \%)$ different codons, respectively (Table S3). However, the codon usage frequencies of the seven species with Escherichia coli were comparatively higher ( 27 different codons). The results indicated that the codon frequency difference between Miscanthus species and Arabidopsis thaliana, Populus trichocarpa and Saccharomyces cerevisiae was the lowest, while was the largest with Escherichia coli. Based on above results, it was optimal to select Saccharomyces cerevisiae, Arabidopsis thaliana and Populus trichocarpa as heterologous expression hosts for Miscanthus and related species. Furthermore, the results indicated that TAG is a different termination codon in usage frequency when comparing the seven assessed plants with the four model species (Table S3).

\section{Source analysis of variation in codon usage} ENC-plot 
277 The ENC and GC3s of the seven analyzed cpDNAs were plotted. It can be seen from Figure 2 278 that the ENC values of most genes were lower than expected values and lie below the standard 279 curve. The results of ENC-plot analysis suggested that codon usage preference of the seven 280 cpDNAs is mainly influenced by natural selection and other factors, while mutation pressure was 281 determined to play only a minor role.

282

283

284

285

286

287

288

289

290

291

292

293

294

295

296

297

298

299

300

301

302

303

304

305

306

307

308

309

310

311

312

313

314

315

316

317

Fi gure 2. ENC-plot (ENC values vs GC3s) for chloroplast genomes of seven Misacanthus and related species. The continuous curve represents the expected curve between GC3s and ENC under random codon usage.

\section{PR2-plot}

PR2-plot is an efficient method to indicate the influence of mutation pressure by investigating the composition of $\mathrm{A}, \mathrm{T}, \mathrm{C}$ and $\mathrm{G}$ at the third nucleotide position of a codon. Our results revealed that the AT-bias is $0.464,0.463,0.463,0.463,0.463,0.465$ and 0.463 for M. floridulus, $M$. giganteus, M. sacchariflorus, M. sinensis, M. transmorrisonensis, Saccharum spontaneumand and Sorghum bicolor, while the GC-bias is $0.512,0.513,0.516,0.515,0.512,0.515$ and 0.518, respectively (Fig 3). Therefore, T/G-bias was observed in all seven assessed species. When considered together, codon usage bias of $\mathrm{A} / \mathrm{T}$ and $\mathrm{G} / \mathrm{C}$ in the seven cp genomes was unbalanced, indicating that the base composition of the seven analyzed cpDNAs is not only influenced by mutation pressure, but also by natural selection.

Figure 3. PR2-plot (A3/(A3+T3) vs G3/(G3+C3)) for chloroplast genomes of seven Misacanthus and related species. The pattern of splashes around the central spot $(A=T, C=G)$ indicate the extent and orientation of the base offset.

\section{Neutrality plot}

The distribution range of GC12 (the mean GC content at the first and second nucleotide positions of a codon) and GC3 is relatively concentrated, in which the range of GC12 is 0.3272 to 0.5469 , and the range of GC3 is 0.179 to 0.512 (Fig 4). No significant correlation was found for GC1 with GC2 $(\mathrm{r} 1=0.157, \mathrm{r} 2=0.168, \mathrm{r} 3=0.128, \mathrm{r} 4=0.127, \mathrm{r} 5=0.161, \mathrm{r} 6=0.155, \mathrm{r} 7=0.140), \mathrm{GC} 1$ with GC3 ( $\mathrm{r} 8=0.092, \mathrm{r} 9=0.079, \mathrm{r} 10=0.055, \mathrm{r} 11=0.049, \mathrm{r} 12=0.100, \mathrm{r} 13=0.242, \mathrm{r} 14=0.202)$ and $\mathrm{GC} 2$ with GC3 (r14=0.054, r14=0.053, r15=-0.014, r16=-0.020, r17 $=0.063, \mathrm{r} 18=-0.032, \mathrm{r} 19=-0.032$ ), which suggested mutation pressure only contributes a minor role in the codon usage preference. In addition, the regression coefficient (slope of neutrality plot) was 0.006 to 0.198 , indicating that the correlation between GC12 and GC3 is not significant, and the composition of the first two bases may be different from the third base of the codon. These results demonstrated that the codon usage patterns of cp coding sequences in the seven species are mainly affected by natural selection.

Figure 4. Neutrality plot (GC12 vs GC3) for chloroplast genomes of seven Misacanthus and related species. The solid line represents the regression line.

Peer) reviewing PDF | (2021:03:58914:3:1:NEW 11 Aug 2021) 


\section{Correspondence analysis (COA)}

320

321

322

323

324

325

326

327

328

329

330

331

332

333

334

335

336

337

338

339

340

341

342

343

344

345

346

347

348

349

350

351

352

353

354

355

356

357

Correspondence analysis is used to explore the variations of codon usage among the analyzed cpDNAs. In the current study, RSCU-based COA was used to compare the usage patterns of 59 codons, which produced a series of orthogonal axes, reflecting the trend of change of codon usage in the seven Miscanhus and related plant species cpDNAs. The first four axes accounted for $36.2 \%, 38.2 \%, 38.5 \%, 38.3 \%, 36.8 \%, 40.7 \%$ and $40.9 \%$ of the overall changes, while the first axis proportion to $14.3 \%, 15.9 \%, 15.8 \%, 15.8 \%, 14.6 \%, 15.6 \%$ and $9.7 \%$ of the total variation in seven species respectively (Table 3 ). Axis 1 , responsible for $10 \%$ of total variation, was the main source of variation, indicating that the codon usage was influenced by multiple factors. In addition, the relationship between axis 1 and axis 2 was visualized to explore the effects of GC content on codon usage bias (Fig 5). Genes with different GC content are plotted as different colors, red with $\mathrm{GC} \%<45 \%$ and blue with $45 \% \leqslant \mathrm{GC} \%<60 \%$ (Fig 5). In order to determine the factors leading to gene dispersion along axis 1 and axis 2 , the correlation index were computed on axis 1 with CAI, CBI, Fop, GC3, GC and L_aa (Table 3). As can be seen from the results in Table 3, axis 1 for M. floridulus, M. sacchariflorus, M. sinensis, $M$. transmorrisonensis, Saccharum spontaneum and Sorghum bicolor possessed a remarkable correlation with GC3s $(\mathrm{p} \leqslant 0.01)$, which indicated the base composition stemming from mutation pressure was the main factor impacting codon usage preference.

Table 3 Correlation analysis of axis 1 and codon usage index of cp genomes of seven Misacanthus and related species (the T/C/A/G content at the third codon position of synonymous codons; codon adaptation index: CAI; codon bias index: CBI; frequency of optimal codons: Fop; the GC content at the third codon position of synonymous codons: GC3s; the GC content at the three position of synonymous codons: GC; total number of amino acids: L_aa)

Figure 5. Visualization of the first two axes from the correspondence analysis based on RSCU values for chloroplast genomes of seven Misacanthus and related species. Genes with different GC content are plotted as different colors, red with $\mathrm{GC} \%<45 \%$ and blue with $45 \% \leqslant \mathrm{GC} \%<60 \%$.

\section{Discussion}

The study compared the codon usage patterns of the five Miscanthus species and two related species. The findings reported here will help to improve our understanding of evolution analysis and the optimization of codon components suitable for gene expression. During the evolutionary processes, specific codon usage patterns were obtained to adapt to the diverse factors including origin, evolution, natural selection and mutation pressure. In addition, analyzing the source of variation in genomic codon usage, the pattern of codon bias and the codon frequency could provide insights into optimization of the codons of heterologous genes and selection of appropriate heterologous expression hosts. Therefore, the research will be of great significance to the study of genetic engineering and genetic evolution.

Peer] reviewing PDF | (2021:03:58914:3:1:NEW 11 Aug 2021) 
358

359

360

361

362

363

364

365

366

367

368

369

370

371

372

373

374

375

376

377

378

379

380

381

382

383

384

385

386

387

388

389

390

391

392

393

394

395

396

397
Our analysis of base composition of codons revealed that the CDSs of the seven Miscanthus and related cpDNAs analyzed tended to use an A/T codon, which was consistent with the results of Zhang et al. (2012) on the 23 Poaceae cpDNAs that this previous study analyzed. According to a previous study, the GC3 values of dicotyledonous plants is often less than $50 \%$ (codon use prefers $\mathrm{A} / \mathrm{T}$ ), which is different from monocotyledonous plants with high GC3 values (GC3 values $>50 \%$, showing that codon use prefers G/C) (Murray et al. 1989). The results of RSCU value analysis showed an A/T codon usage bias in the cpDNAs of the seven analyzed species, which was consistent with the patterns in most higher plants (Shang et al. 2011). According to neutral evolution theory, the effects of mutation pressure and natural selection on the variation of the third base of codon are neutral or nearly neutral (Sharp et al. 1993). The study of Kawabe et al. (2003) showed that when codon use is affected by natural selection, GC3 values tend to be distributed in a small range and there is no significant correlation between GC12 and GC3 (Kawabe et al. 2003). The neutrality plot in this study revealed a weak correlation between $\mathrm{GC} 12$ and $\mathrm{GC} 3$ and the composition of the first two bases were different from the third base of the codon, which demonstrated that the codon usage patterns of the seven cpDNAs analyzed are mainly influenced by natural selection. This result is consistent with the codon usage of cpDNAs of many species, such as Oryza sativa (Liu et al. 2003), Zea mays (maize) (Liu et al. 2009), Triticum aestivum (Liu \& Xue 2005) and Euphorbiaceae (Wang et al. 2020). In addition, combining the results of ENC-plot, PR2-plot and COA analysis suggested that the codon usage bias of the seven cpDNAs were affected by multiple factors, including mutation pressure, base composition and gene length, which the dominant influencing factor was natural selection. This results was consistent with the analysis in Poaceae (Zhang et al. 2012), Populus alba (Zhou et al. 2008) and Euphorbiaceae (Wang et al. 2020).

The cpDNAs of the seven Miscanthus and related plants assessed here are highly conserved and share a total of 21 high-frequency codons. In addition, 4 to 11 codons were determined to be the optimal codons in each species, while no common optimal codon was defined in the seven representative species. These results of high frequency codons and optimal codons are not only beneficial for codon optimization, but also promote further understanding of the relationship between gene expression and codon usage preference. In higher plants, the main obstacle to applying cp transformation to more species and especially, to other important crops is the limitation of available tissue culture systems and regeneration protocols (Ruf et al. 2001). Considering the differences of codon usage bias between the seven cpDNAs analyzed in this study and heterologous expression hosts, codon usage frequencies were analyzed to select the suitable host system. Based on the results, it was suggested to select Saccharomyces cerevisiae, Arabidopsis thaliana and Populus trichocarpa as heterologous expression hosts for Miscanthus and related crops, which possesse a little difference in codon usage frequency with the seven plants.

This study conducted a comprehensive comparative analysis on codon usage patterns at the cpDNA-wide level of seven Miscanthus and related species. These results will improve our 
398

399

400

401

402

403

404

405

406

407

408

409

410

411

412

413

414

415

416

417

418

419

420

421

422

423

424

425

426

427

428

429

430

431

432

433

434

435

436

437

438

439

440

understanding on evolution analysis, the selection of appropriate heterologous expression hosts and the optimization of codon components suitable for gene expression, finally provide a theoretical basis for building a stable and efficient gene expression system in Miscanthus or other crops.

\section{Conclusions}

The codon usage patterns of cp genomes of the five Miscanthus and two related species were compared and systematically analyzed for the first time. The results of codon usage bias and RSCU analysis indicated that the seven representative species prefer the use of $\mathrm{A} / \mathrm{T}$ bases and A/T-ending codons. In addition, 21 common high-frequency codons and 4 to11 optimal codons were elevated in their frequency in the seven cpDNAs. Furthermore, the analysis of codon usage frequencies between the seven representative species and four model organisms suggested that Arabidopsis thaliana, Populus trichocarpa and Saccharomyces cerevisiae could be considered as appropriate heterologous expression hosts for Miscanthus and related species. Finally, when considered together, the results of ENC-plot, PR2-plot and neutrality analysis revealed that the codon usage pattern of the seven cpDNAs analyzed here were influenced by multiple factors, which the dominant influencing factor was natural selection. These results presented in this study potentially provide not only important reference information for evolutionary analysis, but also potentially provide additional important insights for the improvement in the expression efficiency of exogenously introduced genic sequences in transgenic research by codon optimization.

\section{References}

Amiryousefi A, Hyvönen J, and Poczai PJPo. 2018. The chloroplast genome sequence of bittersweet (Solanum dulcamara): Plastid genome structure evolution in Solanaceae. 13:e0196069.

Anue MR, Xueli S, Chunzhen C, Zhongxiong LJPD, and Pests. 2019. Analysis of codon usage pattern of banana basic secretory protease gene. 10:1-9.

Barling A, Swaminathan K, Mitros T, James BT, Morris J, Ngamboma O, Hall MC, Kirkpatrick J, Alabady M, and Spence AKJBg. 2013. A detailed gene expression study of the Miscanthus genus reveals changes in the transcriptome associated with the rejuvenation of spring rhizomes. 14:1-16.

Chae WB, Hong SJ, Gifford JM, Rayburn AL, Sacks EJ, and Juvik JAJGB. 2014. Plant morphology, genome size, and SSR markers differentiate five distinct taxonomic groups among accessions in the genus $M$ iscanthus. 6:646-660.

Chen C, Zheng Y, Liu S, Zhong Y, Wu Y, Li J, Xu L-A, and Xu MJP. 2017. The complete chloroplast genome of Cinnamomum camphora and its comparison with related Lauraceae species. 5:e3820.

Cheng H, Li J, Zhang H, Cai B, Gao Z, Qiao Y, and Mi LJP. 2017. The complete chloroplast genome sequence of strawberry (Fragariax ananassa Duch.) and comparison with related species of Rosaceae. 5:e3919.

Clark LV, Stewart JR, Nishiwaki A, Toma Y, Kjeldsen JB, Jørgensen U, Zhao H, Peng J, Yoo $\mathrm{JH}$, and Heo KJJoEB. 2015. Genetic structure of Miscanthus sinensis and Miscanthus

Peer) reviewing PDF | (2021:03:58914:3:1:NEW 11 Aug 2021) 
441

442

443

444

445

446

447

448

449

450

451

452

453

454

455

456

457

458

459

460

461

462

463

464

465

466

467

468

469

470

471

472

473

474

475

476

477

478

479

480

481

482

483

484

485

486

487

488

489

490

491

sacchariflorus in Japan indicates a gradient of bidirectional but asymmetric introgression. 66:4213-4225.

Ge C, Liu X, Liu S, Xu J, Li H, Cui T, Yao Y, Chen M, Yu W, and Chen CJPMBR. 2017. Miscanthus sp.: genetic diversity and phylogeny in China. 35:600-610.

Havaux M, Lütz C, and Grimm BJPP. 2003. Chloroplast membrane photostability in chlPtransgenic tobacco plants deficient in tocopherols. 132:300-310.

Jarvis P, and López-Juez EJNRMCB. 2013. Biogenesis and homeostasis of chloroplasts and other plastids. 14:787-802.

Kawabe A, Miyashita NTJG, and systems g. 2003. Patterns of codon usage bias in three dicot and four monocot plant species. 78:343-352.

Khodakovskaya M, McAvoy R, Peters J, Wu H, and Li YJP. 2006. Enhanced cold tolerance in transgenic tobacco expressing a chloroplast $\omega-3$ fatty acid desaturase gene under the control of a cold-inducible promoter. 223:1090-1100.

Kwak S-Y, Lew TTS, Sweeney CJ, Koman VB, Wong MH, Bohmert-Tatarev K, Snell KD, Seo JS, Chua N-H, and Strano MSJNn. 2019. Chloroplast-selective gene delivery and expression in planta using chitosan-complexed single-walled carbon nanotube carriers. 14:447-455.

Liu H, He R, Zhang H, Huang Y, Tian M, and Zhang J. 2009. Analysis of synonymous codon usage in Zea mays. Molecular Biology Reports 37:677-684. 10.1007/s11033-009-9521-7

Liu Q-P, Tan J, and Xue Q-ZJYcxbAgS. 2003. Synonymous codon usage bias in the rice cultivar 93-11 (Oryza sativa L. ssp. indica). 30:335-340.

Liu Q, Feng Y, Dong H, and Xue QJPS. 2004. Synonymous codon usage bias in Oryza sativa. 167:101-105.

Liu Q, and Xue QJJog. 2005. Comparative studies on codon usage pattern of chloroplasts and their host nuclear genes in four plant species. 84:55-62.

Murray EE, Lotzer J, and Eberle MJNAR. 1989. Codon usage in plant genes. 17:477-498.

Nielsen AZ, Mellor SB, Vavitsas K, Wlodarczyk AJ, Gnanasekaran T, Perestrello Ramos H de Jesus M, King BC, Bakowski K, and Jensen PEJTPJ. 2016. Extending the biosynthetic repertoires of cyanobacteria and chloroplasts. 87:87-102.

Pan L-L, Wang Y, Hu J-H, Ding Z-T, and Li CJJotb. 2013. Analysis of codon use features of stearoyl-acyl carrier protein desaturase gene in Camellia sinensis. 334:80-86.

Plotkin JB, and Kudla GJNRG. 2011. Synonymous but not the same: the causes and consequences of codon bias. 12:32-42.

Pop C, Rouskin S, Ingolia NT, Han L, Phizicky EM, Weissman JS, and Koller DJMsb. 2014. Causal signals between codon bias, mRNA structure, and the efficiency of translation and elongation. 10:770.

Quax TE, Claassens NJ, Söll D, and van der Oost JJMc. 2015. Codon bias as a means to finetune gene expression. 59:149-161.

Ravi V, Khurana J, Tyagi A, Khurana PJPS, and Evolution. 2008. An update on chloroplast genomes. 271:101-122.

Romero H, Zavala A, and Musto HJNar. 2000. Codon usage in Chlamydia trachomatis is the result of strand-specific mutational biases and a complex pattern of selective forces. 28:2084-2090.

Ruf S, Hermann M, Berger IJ, Carrer H, and Bock RJNb. 2001. Stable genetic transformation of tomato plastids and expression of a foreign protein in fruit. 19:870-875.

Shang M, Liu F, Hua J, and Wang KJSAS. 2011. Analysis on codon usage of chloroplast genome of Gossypium hirsutum. 44:245-253.

Sharp PM, and Li W-HJJome. 1986. An evolutionary perspective on synonymous codon usage in unicellular organisms. 24:28-38.

Sharp PM, Stenico M, Peden JF, and Lloyd ATJBST. 1993. Codon usage: mutational bias, translational selection, or both? 21:835-841.

Peer) reviewing PDF | (2021:03:58914:3:1:NEW 11 Aug 2021) 
492

493

494

495

496

497

498

499

500

501

502

503

504

505

506

507

508

509

510

511

512

513

514

515

516

517

518

519

520

521

522

523

524

525

526

527

528

529

530

531

532

533

534

535

536

537

538

539

540

541

542

Sheng J, Hu X, Zeng X, Li Y, Zhou F, Hu Z, Jin S, and Diao Y. 2016. Nuclear DNA content in Miscanthus sp. and the geographical variation pattern in Miscanthus lutarioriparius. Sci Rep 6:34342. 10.1038/srep34342

Sheng J, Yan M, Wang J, Zhao L, Zhou F, Hu Z, Jin S, and Diao Y. 2021. The complete chloroplast genome sequences of five Miscanthus species, and comparative analyses with other grass plastomes. Industrial Crops and Products 162. 10.1016/j.indcrop.2021.113248

Sheng J, Zheng X, Wang J, Zeng X, Zhou F, Jin S, Hu Z, and Diao Y. 2017. Transcriptomics and proteomics reveal genetic and biological basis of superior biomass crop Miscanthus. Scientific Reports 7. 10.1038/s41598-017-14151-z

Sueoka NJG. 1999. Translation-coupled violation of Parity Rule 2 in human genes is not the cause of heterogeneity of the DNA G+ C content of third codon position. 238:53-58.

Sueoka NJJome. 1995. Intrastrand parity rules of DNA base composition and usage biases of synonymous codons. 40:318-325.

Sueoka NJPotNAoS. 1988. Directional mutation pressure and neutral molecular evolution. 85:2653-2657.

Tuller T, Waldman YY, Kupiec M, and Ruppin EJPotNAoS. 2010. Translation efficiency is determined by both codon bias and folding energy. 107:3645-3650.

Vermerris W. 2008. Miscanthus: genetic resources and breeding potential to enhance bioenergy production. Genetic improvement of bioenergy crops: Springer, 295-308.

Wang Z, Xu B, Li B, Zhou Q, Wang G, Jiang X, Wang C, and Xu Z. 2020. Comparative analysis of codon usage patterns in chloroplast genomes of six Euphorbiaceae species. PeerJ 8:e8251. 10.7717/peerj.8251

Wright FJG. 1990. The 'effective number of codons' used in a gene. 87:23-29.

Wu Y, Zhou N, Ni X, Okoye CO, Wang Y, Li X, Gao L, Zhou G, Jiang JJIC, and Products. 2021. Developing a long-term and powerful in vitro culture and Agrobacterium-mediated transformation system for Miscanthus sinensis (Poaceae). 161:113190.

Xiang H, Zhang R, Butler III RR, Liu T, Zhang L, Pombert J-F, and Zhou ZJPo. 2015. Comparative analysis of codon usage bias patterns in microsporidian genomes. 10:e0129223.

Yan M, Zhao X, Zhou J, Huo Y, Ding Y, and Yuan ZJljoms. 2019. The complete chloroplast genomes of Punica granatum and a comparison with other species in Lythraceae. 20:2886.

Zhang J, Yan J, Zhang Y, Ma X, Bai S, Wu Y, Dao Z, Li D, Zhang C, and Zhang YJPo. 2013. Molecular insights of genetic variation in Erianthus arundinaceus populations native to China. 8:e80388.

Zhang R, Zhang L, Wang W, Zhang Z, Du H, Qu Z, Li X-Q, and Xiang HJljoms. 2018. Differences in codon usage bias between photosynthesis-related genes and genetic system-related genes of chloroplast genomes in cultivated and wild solanum species. 19:3142.

Zhang WJ, Zhou, J., Li, Z. F., Wang, L., Gu, X., \& Zhong, Y. 2007. Comparative analysis of codon usage patterns among mitochondrion, chloroplast and nuclear genes in Triticum aestivum L. . Journal of Integrative Plant Biology 49:246-254.

Zhang Y, Nie X, Jia X, Zhao C, Biradar SS, Wang L, Du X, and Weining SJAJoB. 2012. Analysis of codon usage patterns of the chloroplast genomes in the Poaceae family. 60:461-470.

Zhou M, Long W, and Li XJJoFR. 2008. Analysis of synonymous codon usage in chloroplast genome of Populus alba. 19:293-297.

Zhou M, Tong C, Shi JJJoG, and Genomics. 2007. Analysis of codon usage between different poplar species. 34:555-561.

Peer) reviewing PDF | (2021:03:58914:3:1:NEW 11 Aug 2021) 


\section{Table $\mathbf{1}$ (on next page)}

Genomic features of chloroplast genomes of the seven Miscanthus and related species

the total number of amino acids: L_aa ; the GC content at the first, second and third codon positions: GC1, GC2and GC3; average GC at three locations: GC123 
1 Table 1 Genomic features of chloroplast genomes of the seven Miscanthus and related species (the total number of amino acids: L_aa ; the GC content at 2 the first, second and third codon positions: GC1, GC2and GC3; average GC at three locations: GC123).

\begin{tabular}{llllllll}
\hline Parameters & $\begin{array}{l}\text { Miscanthus } \\
\text { floridulus }\end{array}$ & $\begin{array}{l}\text { Miscanthus } \\
\text { giganteus }\end{array}$ & $\begin{array}{l}\text { Miscanthus } \\
\text { sacchariflorus }\end{array}$ & $\begin{array}{l}\text { Miscanthus } \\
\text { sinensis }\end{array}$ & $\begin{array}{l}\text { Miscanthus } \\
\text { transmorrisonensis }\end{array}$ & $\begin{array}{l}\text { Saccharum } \\
\text { spontaneum }\end{array}$ & $\begin{array}{l}\text { Sorghum } \\
\text { bicolor }\end{array}$ \\
\hline L_aa & 19611 & 19469 & 19508 & 19506 & 19486 & 16553 & 17490 \\
CDSs number (before filting) & 106 & 106 & 122 & 122 & 106 & 76 & 84 \\
CDSs number (after filting) & 65 & 64 & 64 & 64 & 64 & 48 & 52 \\
GC1 & 0.473 & 0.474 & 0.472 & 0.472 & 0.473 & 0.477 & 0.476 \\
GC2 & 0.397 & 0.397 & 0.396 & 0.396 & 0.397 & 0.395 & 0.393 \\
GC3 & 0.312 & 0.311 & 0.311 & 0.311 & 0.311 & 0.302 & 0.303 \\
GC123 & 0.394 & 0.394 & 0.393 & 0.393 & 0.394 & 0.391 & 0.39 \\
\hline
\end{tabular}

3 
Table 2 (on next page)

Optimal codons in chloroplast genomes of the seven Miscanthus and related species 
1 Table 2 Optimal codons in chloroplast genomes of the seven Miscanthus and related species.

\begin{tabular}{lll}
\hline Species & $\begin{array}{l}\text { Optimal codon } \\
\text { numbers }\end{array}$ & Optimal codon \\
\hline $\begin{array}{l}\text { Miscanthus floridulus } \\
\text { Miscanthus giganteus }\end{array}$ & 4 & 'CAC', 'CCA', 'TCA', 'TAG' \\
$\begin{array}{l}\text { Miscanthus sacchariflorus } \\
\text { Miscanthus sinensis }\end{array}$ & 4 & 'TTC', 'CTT', 'CCA', 'AGG', 'TCA', 'TGA' \\
& 11 & 'GCC', 'CTT', 'AGG', 'ACG' \\
$\begin{array}{l}\text { Miscanthus transmorrisonensis } \\
\text { Saccharum spontaneum }\end{array}$ & 4 & 'GCC', 'TTC', 'GGC', 'ATA', 'CTA', 'AGA', \\
Sorghum bicolor & 4 & 'AGG', 'TCT', 'ACC', 'ACG', 'GTC' \\
& 8 & 'CTT', 'CCA', 'TCA', 'TGA' \\
& & 'CTT', 'CCA', 'TCA', 'TAG' \\
\hline
\end{tabular}

2 


\section{Table 3 (on next page)}

Correlation analysis of axis 1 and codon usage index of chloroplast genomes of seven Misacanthus and related species

the $T / C / A / G$ content at the third codon position of synonymous codons; codon adaptation index: $\mathrm{CAl}$; codon bias index: $\mathrm{CBI}$; frequency of optimal codons: Fop; the GC content at the third codon position of synonymous codons: GC3s; the GC content at the three position of synonymous codons: GC; total number of amino acids: $L_{-}$aa 
Table 3 Correlation analysis of axis 1 and codon usage index of chloroplast genomes of seven Misacanthus and related species (the T/C/A/G content at the third codon position of synonymous codons; codon adaptation index: CAI; codon bias index: CBI; frequency of optimal codons: Fop; the GC content at the third codon position of synonymous codons: GC3s; the GC content at the three position of synonymous codons: GC; total number of amino acids: L_aa)

\begin{tabular}{|c|c|c|c|c|c|c|c|c|c|c|c|}
\hline Species & T3s & C3s & A3s & G3s & CAI & CBI & Fop & Ne & GC3s & GC & L_aa \\
\hline Miscanthus floridulus & $-0.65 * *$ & $0.507 * *$ & -0.133 & $0.598 * *$ & -0.152 & 0.073 & 0.098 & $0.28 * *$ & $0.639 * *$ & 0.159 & $-0.315 * *$ \\
\hline Miscanthus $x$ giganteus & 0.016 & 0.053 & -0.104 & -0.127 & -0.038 & 0.044 & 0.078 & 0.09 & -0.018 & $0.223 * *$ & 0.057 \\
\hline Miscanthus sacchariflorus & $0.66 * *$ & $-0.463 * *$ & $0.171 *$ & $-0.708 * *$ & $0.176^{*}$ & 0.044 & 0.086 & $-0.43 * *$ & $-0.7 * *$ & -0.154 & 0.118 \\
\hline Miscanthus sinensis & $0.663 * *$ & $-0.469 * *$ & $0.18^{*}$ & $-0.711 * *$ & $0.17 *$ & 0.043 & 0.083 & $-0.423 * *$ & $-0.708 * *$ & -0.154 & 0.123 \\
\hline Miscanthus transmorrisonensis & $0.647 * *$ & $-0.509 * *$ & 0.129 & $-0.604 * *$ & 0.147 & -0.076 & -0.099 & $-0.285 * *$ & $-0.641 * *$ & -0.152 & $0.318 * *$ \\
\hline Saccharum spontaneum & $0.693 * *$ & $-0.479 * *$ & $0.201 *$ & $-0.691 * *$ & 0.193 & 0.02 & -0.012 & $-0.217^{*}$ & $-0.667 * *$ & -0.19 & $0.197 *$ \\
\hline Sorghum bicolor & $0.412 * *$ & $-0.464 * *$ & $0.25 * *$ & $-0.726^{* *}$ & $0.33 * *$ & $0.253 * *$ & $0.252 * *$ & $-0.48 * *$ & $-0.685^{* *}$ & -0.035 & 0.034 \\
\hline
\end{tabular}


Figure 1

Codon content in all protein-coding genes of the seven Miscanthus and related $\mathrm{cp}$ genomes

From left to right: $M$. floridulus, $M$. giganteus, $M$. sacchariflorus, $M$. sinensis, $M$.

transmorrisonensis, Saccharum spontaneum and Sorghum bicolor

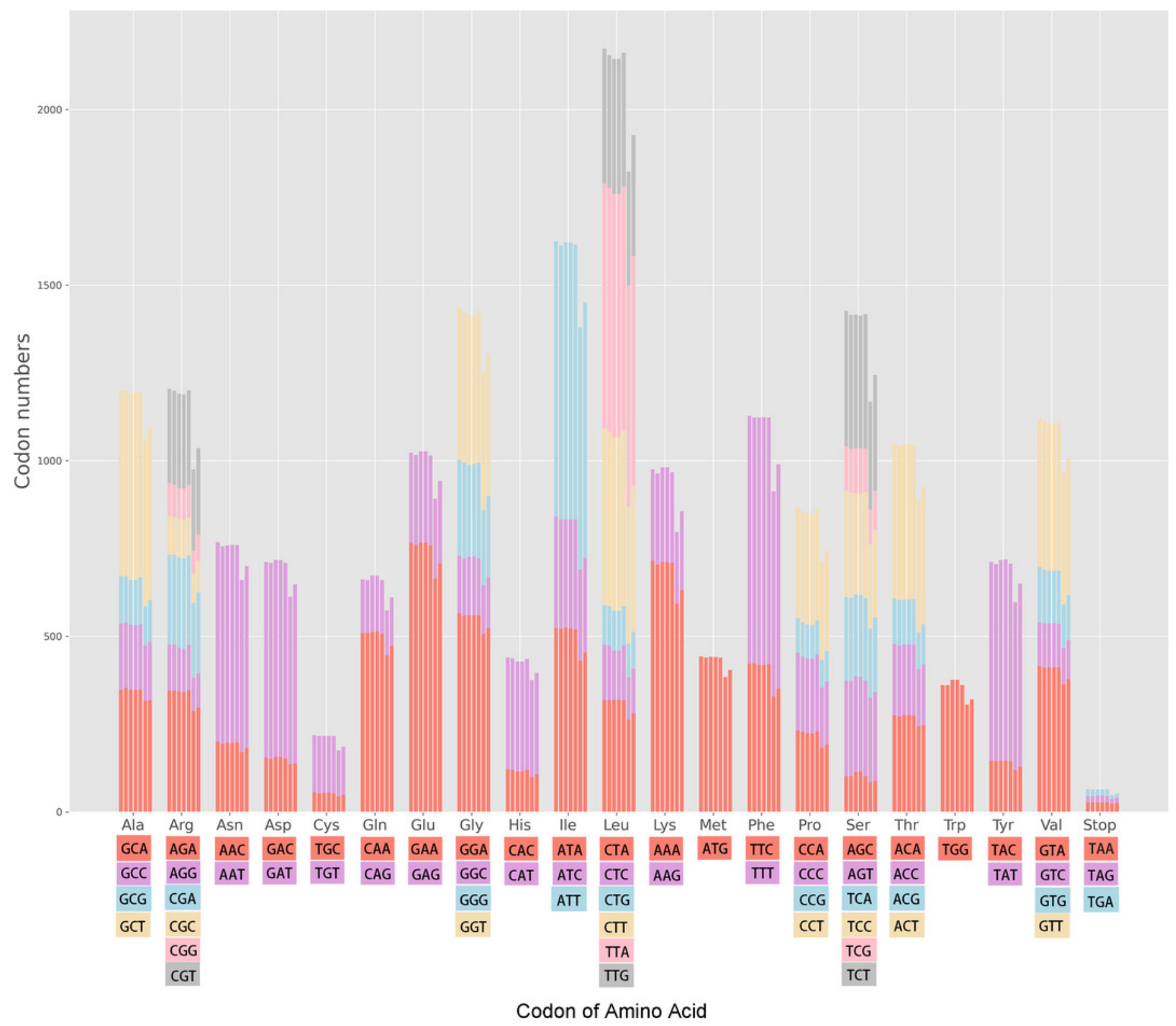


Figure 2

ENc-plot of chloroplast genomes of seven Misacanthus and related species
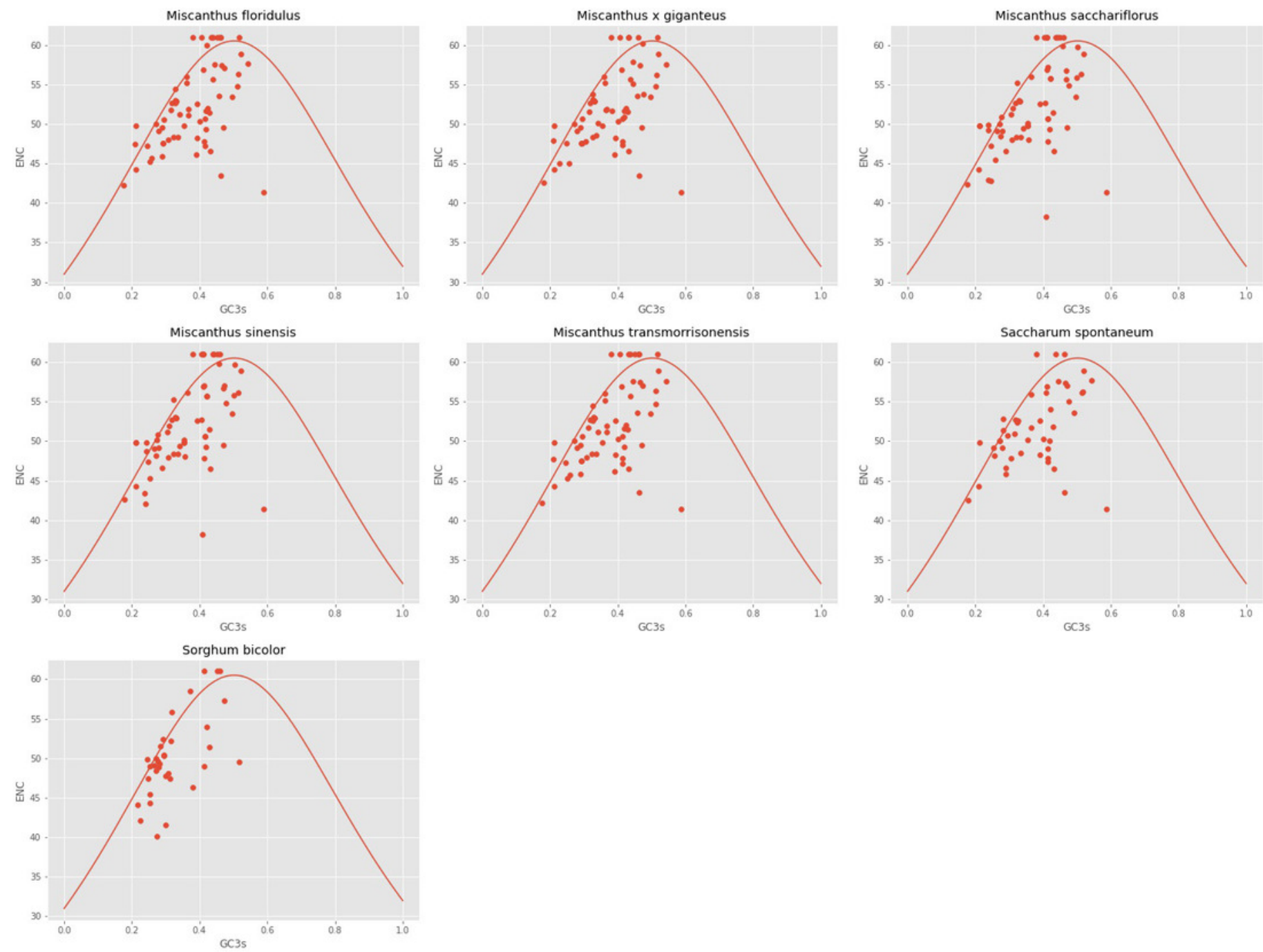
Figure 3

\section{PR2-plot of chloroplast genomes of seven Misacanthus and related species}
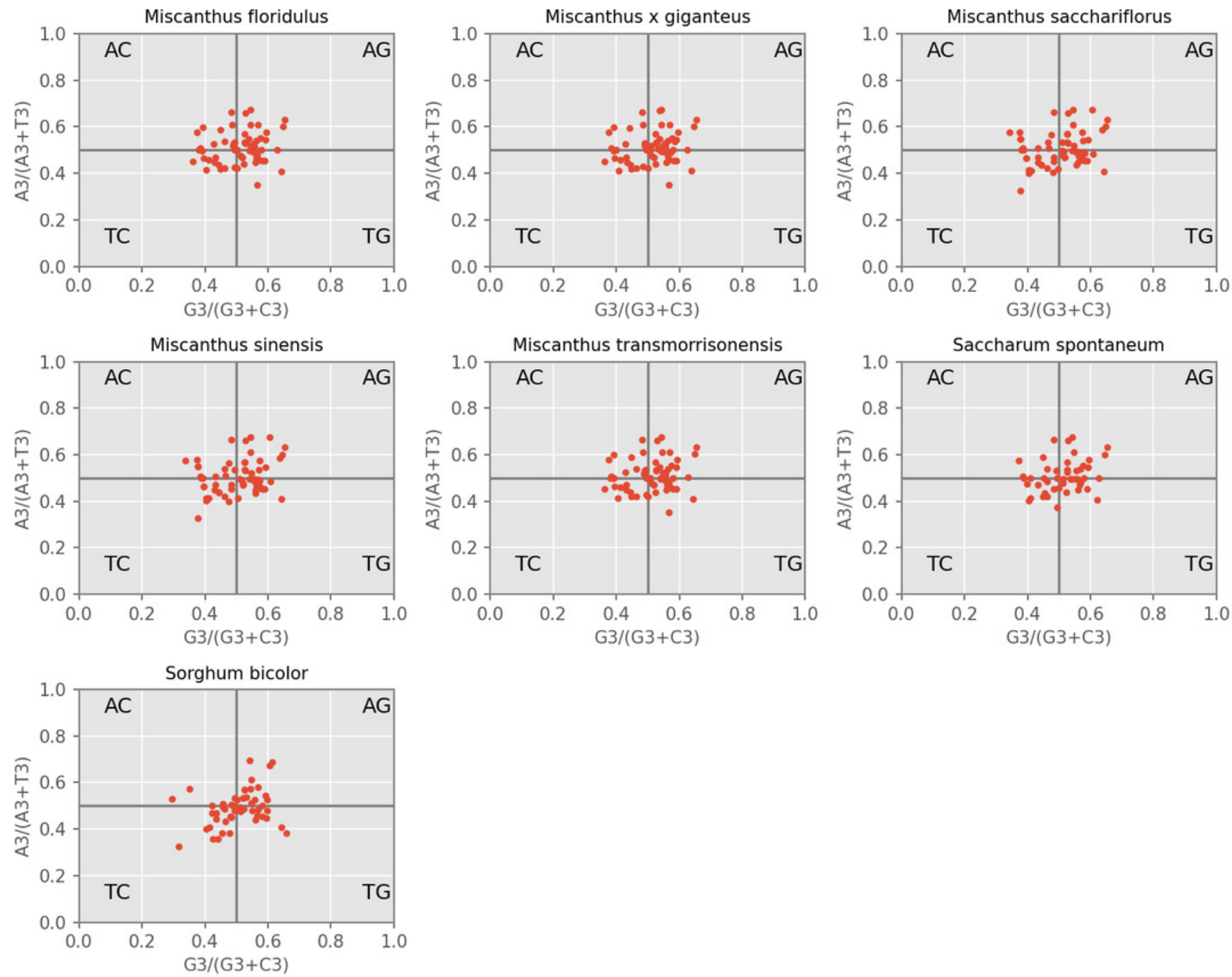
Figure 4

\section{Neutrality plot of chloroplast genomes of seven Misacanthus and related species}
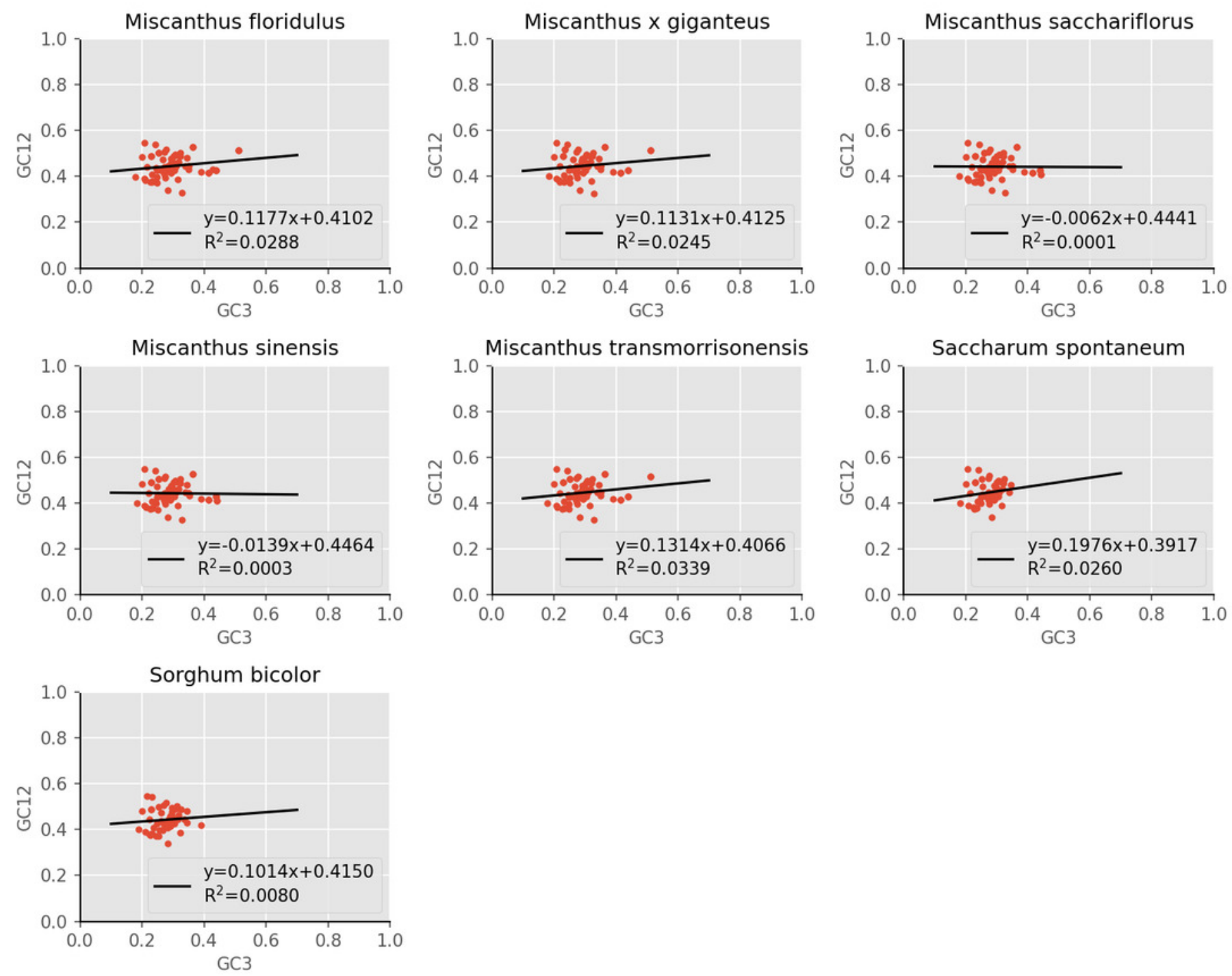
Figure 5

\section{Correspondence analysis of chloroplast genomes of seven Misacanthus and related species}
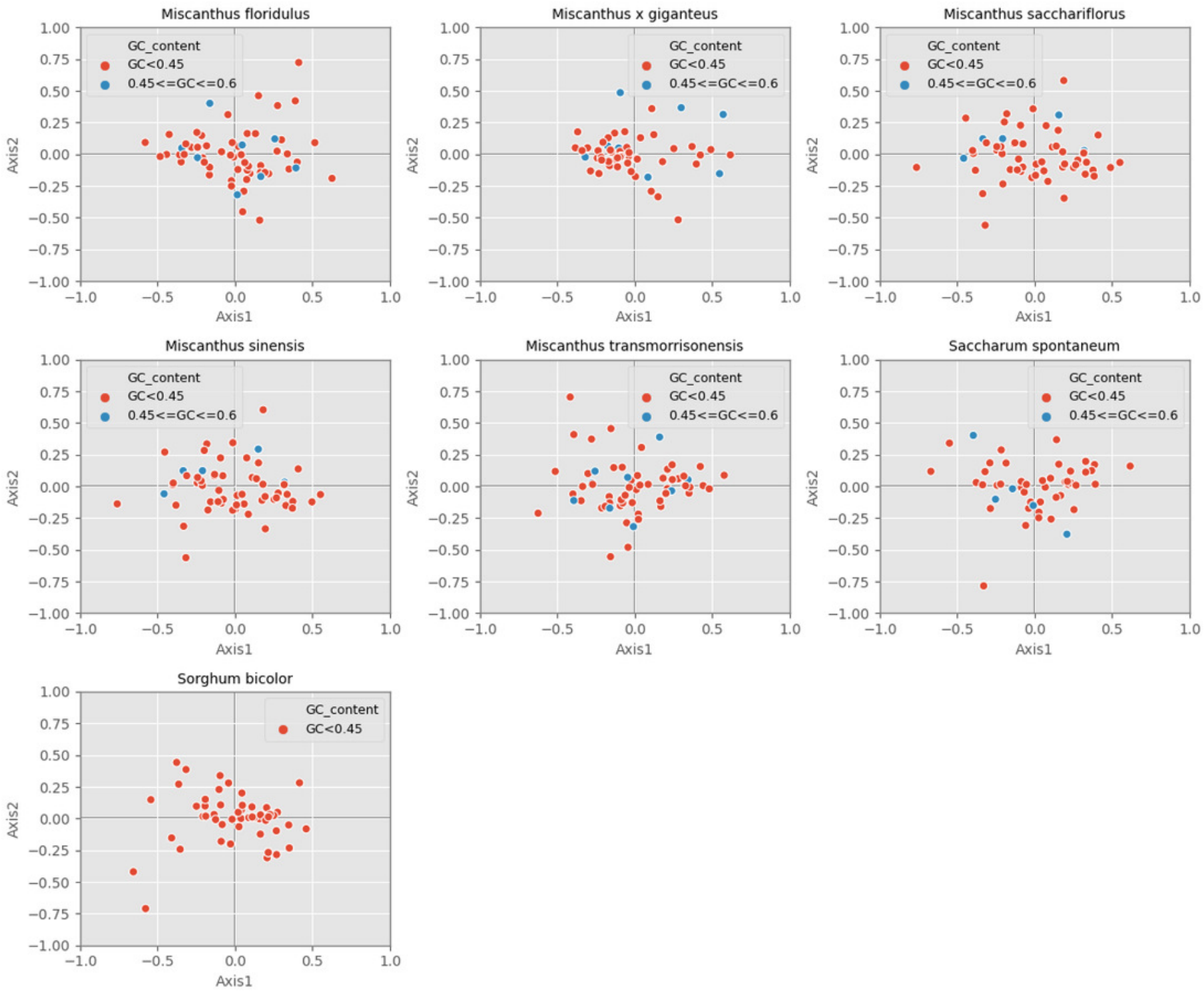